\title{
"Turns" analysis in the physiological evaluation of neuromuscular disorders
}

\author{
H A GARCIA, H S MILNER-BROWN, AND M A FISHER \\ From the Laboratory of EMG and Clinical Neurophysiology, Department of Neurological Sciences, \\ Rush-Presbyterian St Luke's Medical Center, Chicago, USA
}

SUMMARY A previously described technique of automatic analysis of the electromyogram during isometric voluntary contraction has been used to investigate the biceps and triceps muscles of 18 patients with various neuromuscular disorders. Three modifications in the method were made: at each electrode position the data were computed at $10 \%, 20 \%, 30 \%$ and $50 \%$ of the maximum force; the duration of contraction was reduced from $5 \mathrm{~s}$ to $2 \mathrm{~s}$; and the diagnosis was based on analysis of the plots of turns and mean amplitude per turn versus percentage of maximum force. These modifications resulted in increased sensitivity, the ability to evaluate motor unit abnormality at different force levels, and a quantitative assessment of the degree of involvement of antagonist muscles. The data indicate that motor units recruited at lower force levels may be involved preferentially in myopathies, and also that the involvement of pairs of flexor and extensor muscles in neuromuscular disorders is not always uniform.

A method for quantitatively evaluating muscle electrical activity recorded with a needle electrode during voluntary contraction was introduced over a decade ago. ${ }^{2}$ The method involved counting the number of positive and negative potential changes exceeding $100 \mu \mathrm{V}$ ("turns") and their amplitudes when the muscle supported a constant load of two or five $\mathrm{kg}$. Using this method, the number of turns was increased in patients with primary muscle disease ${ }^{2}$ while elevation of mean amplitude has been emphasised as an index of chronic partial denervation. ${ }^{3}$

When a relatively weak muscle supports a standard load, however, the computed turns may be misinterpreted. The muscle activity needed to support this tension will be greater than in a stronger muscle and therefore the recorded turns will also be larger. In order to reduce this effect, it has been suggested that the turns and amplitudes should be computed at a fixed proportion of the maximum force with $30 \%$ giving the best diagnostic yield. ${ }^{4-0}$ Increased turns and decreased mean amplitudes have been characteristic of

Address for reprint requests: Dr MA Fisher, Michael Reese Hospital and Medical Center, 29th Street and Ellis Avenue, Chicago, Illinois, 60616, USA.

Accepted 1 June 1980 myopathies while the reverse has been found in neuropathies.

The single fixed $30 \%$ maximum force would be justified theoretically if all neuromuscular disorders uniformly affected motor units recruited at different force levels. However, since this may not always be the case, we decided to compute the parameters at $10 \%, 20 \%, 30 \%$, and $50 \%$ of maximum force in each subject. This modified procedure has been used to quantitatively analyse the electrical activity recorded from the biceps or triceps or both muscles of 12 normal subjects and 18 patients with various neuromuscular disorders. This paper is a summary of this study with emphasis on the contribution to electrodiagnosis that evolved from this approach.

\section{Methods}

Subjects 12 normal subjects (seven males, five females) age 20 to 45 years with no signs or symptoms of neuromuscular diseases were tested. Eighteen patients (ages 14 to 68 years) were evaluated. Nine had primary muscle disease: limb-girdle dystrophy five, slowly progressive $\mathrm{x}$-linked (Becker) muscular dystrophy (one), myotonic dystrophy (one), polymyositis (one), dermatomyositis (one). Two were thought to have probable muscle disease: (one) patient with 
progressive weakness on steroid therapy, and another with weakness as well as tender muscles associated with scleroderma. Five were considered to have primary neuropathic dysfunction: spinal muscular atrophy type three (Kugelberg-Welander disease) (three), peroneal muscular atrophy (Charcot-Marie-Tooth disease) (one), and peripheral neuropathy associated with chronic renal failure (one) and a lymphoma (one). In one patient, the primary pathology was unclear at the time of study; a 19 year old male with a long history of non-progressive proximal muscle weakness and elevated creatine phosphokinase (CPK). In all subjects, data were obtained from the biceps brachii while in eight, recordings were also made in the triceps brachii of the same arm.

In all patients, standard electrodiagnostic examinations were performed including motor and sensory conduction velocities, and EMG examination including analysis of a series of individual motor units in selected muscles.

Force measurements The isometric force exerted by the biceps or triceps was measured with a force bracket which consisted of a metal bracket with a bonded strain gauge and a strap. The bridge amplifier output was fed into a digital voltmeter and calibrated $(1 \mathrm{mV}=120 \mathrm{mg})$. The force exerted could then be instantly read on the digital voltmeter (in $\mathrm{mv})$ by both subjects and investigator.

Electrical activity The electrical activity was recorded with a bipolar needle electrode (DISA 13K80). The electrical signal was amplified (TECA AA6MKIII set at $500 \mu \mathrm{V} / \mathrm{div}$ ) and then analysed by an action potential ("turns") analyser TECA APA6MK2). The computed number of turns, amplitude, and amplitude/turns ratio per second were instantly displayed on the oscilloscope and printed on a photosensitive paper.

Procedure The subject lay supine on a bed with the elbow flexed at approximately $90^{\circ}$, and the force bracket strapped to the wrist. The subject was then asked to flex the elbow maximally for 1 to $2 \mathrm{~s}$, and the maximum force displayed on the digital voltmeter noted; $10 \%, 20 \%, 30 \%$ and $50 \%$ of the maximum force was then calculated in mv. The bipolar needle electrode was next inserted into the biceps muscle and the subject asked to exert $50 \%$, $10 \%, 20 \%$ and $30 \%$ of the maximum force for 2 s with periods of relaxation between each contraction.

The depth of the needle in the muscle was altered by approximately $5 \mathrm{~mm}$, and the whole procedure repeated. Generally, the needle was inserted at 3 locations 1-1.5 $\mathrm{cm}$ apart. At each insertion the depth of the needle within the muscle was altered two to three times, so that a total of nine sites would be investigated. The position of the force bracket would then be modified for elbow extension and the triceps investigated in a similar manner. The procedure takes 45-60 min depending on the subject.

Data analysis The number of turns, cumulative amplitude, and amplitude/turns ratio were computed for $2 \mathrm{~s}$ at each force level and printed on the photo- sensitive paper. The average of the nine values at each of the $10 \%, 20 \%, 30 \%$ and $50 \%$ maximum force levels was then calculated. Turns (and amplitude) against $\%$ maximum force were then plotted.

The procedure is harmless and all of the normal subjects gave informed consent. The patients were studied as part of their routine electrodiagnostic evaluation.

\section{Results}

The difference between the present procedure and the original method as well as the recent modification are as follows: (1) the electrical activity from the muscle was recorded at $10 \%$, $20 \%, 30 \%$ and $50 \%$ maximum force instead of at fixed load or at a fixed percentage of maximum force, (2) the analysis was for only $2 \mathrm{~s}$ of voluntary activity instead of $5 \mathrm{~s}$, (3) data analysis was based on the evaluation of turns and amplitude against \% maximum force, and (4) bipolar rather than concentric needle electrodes were used. The results obtained from normal subjects and patients with neuromuscular disorders will be presented. Circumstances in which the advantages of the present modifications are evident will be emphasised.

\section{Control subjects}

In males, the maximal tension ranged between 21 to $28 \mathrm{~kg}$ while for females the range was 14 to $19 \mathrm{~kg}$ (cf the $17-38 \mathrm{~kg}$ range reported by Fuglsang-Frederickson and Mansson). Data was recorded at $10,20,30$, and $50 \%$ levels of these maximum force.

The number of turns were reproduced with increasing difficulty if the analysis time was greater than 3 s. At $50 \%$, a slight tremor might appear, and the number of turns recorded might even be less than at $30 \%$. In order to avoid these problems, turn counts were obtained during a 2 s period after stable conditions for the appropriate force level were reached. Using this procedure, a steady tension could be obtained for every trial, and the subjects did not complain of discomfort or fatigue.

In several subjects, turns counts were obtained not only in different areas (see methods) but also three times in the same area. The variance was approximately 10 times greater comparing data recorded in the former manner in contrast to the latter. Reproducible data was therefore obtained with a $2 \mathrm{~s}$ recording period and data from different sites was considered more important for sampling than repeated information from the same site.

Figure 1 is representative normal data at 10 , 


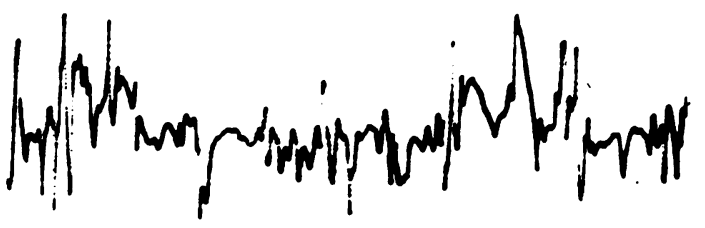

AG53日 T1134 MESE

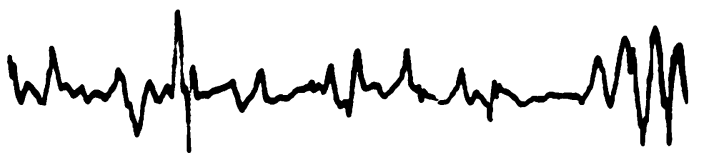

A9255 TBES9 MESE
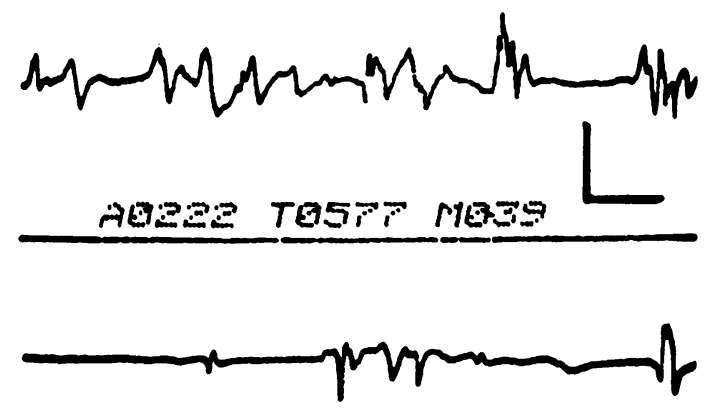

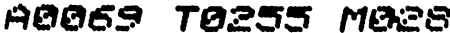

Fig 1 Shows a typical print out of motor unit activity and computed parameters for a single electrode location. The upper records represent a sample EMG activity of $100 \mathrm{~ms}$ duration, although the analysis was over $2 \mathrm{~s}$ periods. The lower traces are computed values over the 2 s period of Amplitude

$$
\text { (A), Turns (T), } \frac{\text { Amplitude }}{\text { Turns }} \text { ratio ( } M \text { or } A / T \text { ratio). }
$$

The 4 records, bottom to top are at 10\%, 20\%, 30\% and $50 \%$ of this subject's maximum force of $24 \mathrm{~kg}$. Calibration $500 \mu \mathrm{V} 10 \mathrm{~ms}$.

20,30 , and $50 \%$ of the maximal force. Note the increasing EMG activity with increasing force associated with increasing cumulative amplitude (A), turns ( $\mathrm{T}$ ), and mean amplitude/turn ratio (A/T). Because of the gain setting of the amplifiers, the actual amplitudes were one-half those computed by the action potential analyser.

Figure $2 \mathrm{~A}$ is a plot of the control mean values for turns with standard deviations (SD). Figure 2B presents comparable information for cumu- lative amplitudes. Females have somewhat greater number of turns and amplitudes than males at corresponding levels of force. The plot
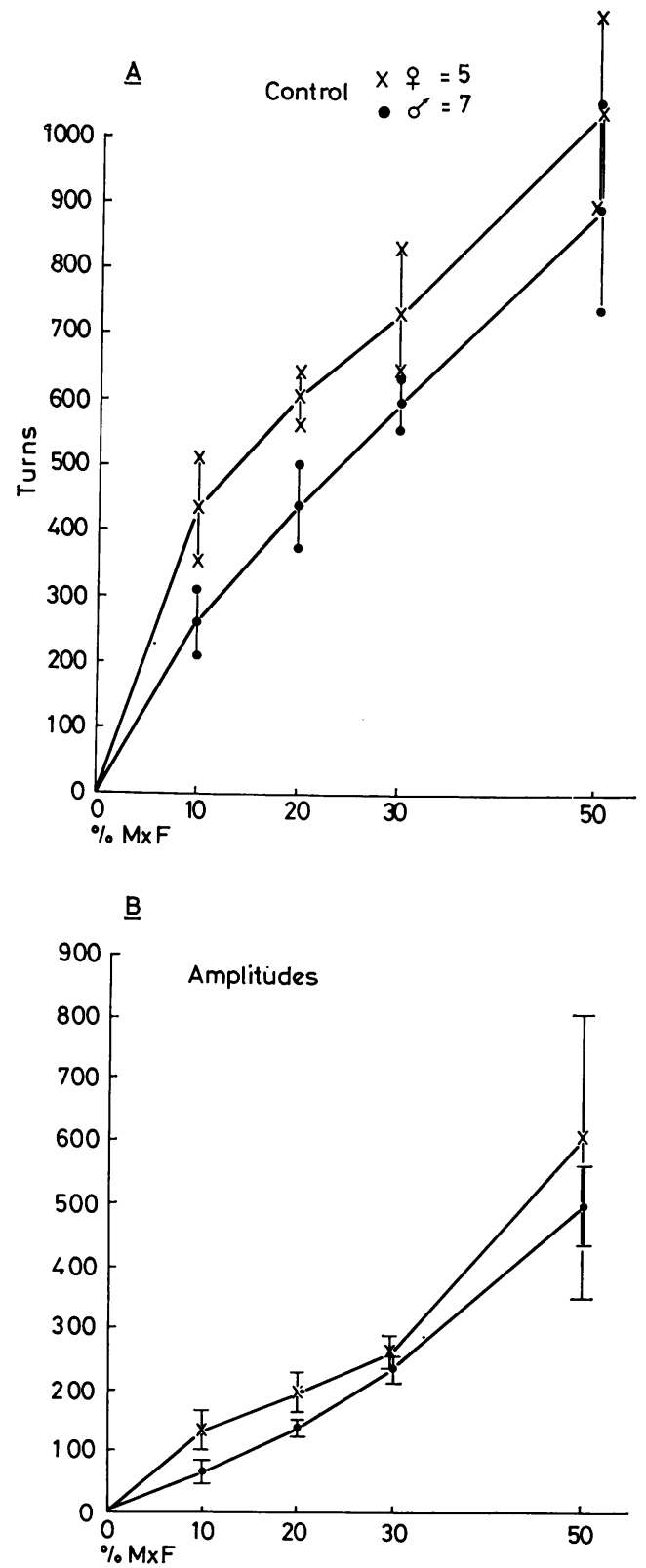

Fig 2 Plot of (A) turns, (B) amplitude as a function of \% maximum force exerted by the biceps brachii during elbow flexion. The values are the mean $\pm S D$ recorded from seven males and five females. In each subject, the electrical parameters were computed for nine electrode locations. 
Table 1 Average mean amplitude per turn

\begin{tabular}{lllllllll}
\hline & $M$ & Biceps & Brachii & & $M$ & Triceps & Brachii \\
\hline Load & $10 \%$ & $20 \%$ & $30 \%$ & $50 \%$ & $10 \%$ & $20 \%$ & $30 \%$ & $50 \%$ \\
Subjects & 12 & 12 & 12 & 12 & 10 & 10 & 10 & 10 \\
Mean amplitude (mV) & 0.133 & 0.154 & 0.176 & 0.283 & $0 \cdot 141$ & $0 \cdot 177$ & $0 \cdot 194$ & 0.296 \\
SD & 0.025 & 0.030 & 0.038 & 0.063 & 0.031 & 0.040 & 0.048 & $0 \cdot 115$ \\
\hline
\end{tabular}

of turns versus relative force is more linear than that for amplitude. The SD's for cumulative amplitudes at $50 \%$ maximum force are proportionally quite large. The average mean amplitude per turn is shown in table 1 .

Comparable data were obtained in 10 control studies in the triceps brachii. The range of maximum force was 6 to $18 \mathrm{~kg}$. The turns and cumulative amplitudes for similar proportional force were somewhat higher in females than males. The data for turns related to percentage maximum force was more linear and the SD's at the higher percentages of force lower than for cumulative amplitudes. The range of control values for turns is shown in fig 4 and $A / T$ 's in table 1.

\section{Patients}

The turns and $\mathrm{A} / \mathrm{T}$ data for the patients are shown in table 2. If the turns were increased or decreased, this was true at all levels of force,

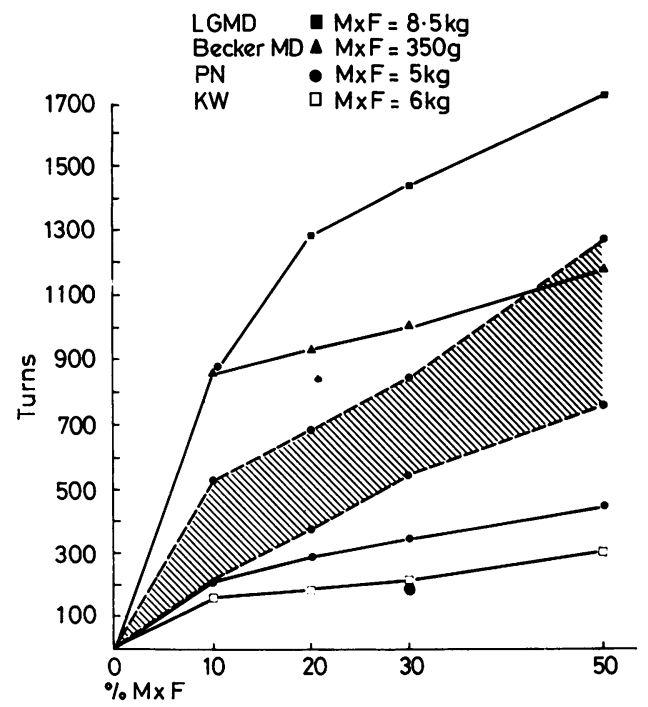

Fig 3 A plot of turns against \% maximum force of 12 control subjects (hatched area), two patients with primary muscle disease and two patients with neurogenic involvement. LGMD, limb girdle muscular dystrophy; Becker MD, Becker muscular dystrophy; $P N$, peripheral neurcpathy; $K W, K u g e l b e r g-$ Welander disease.

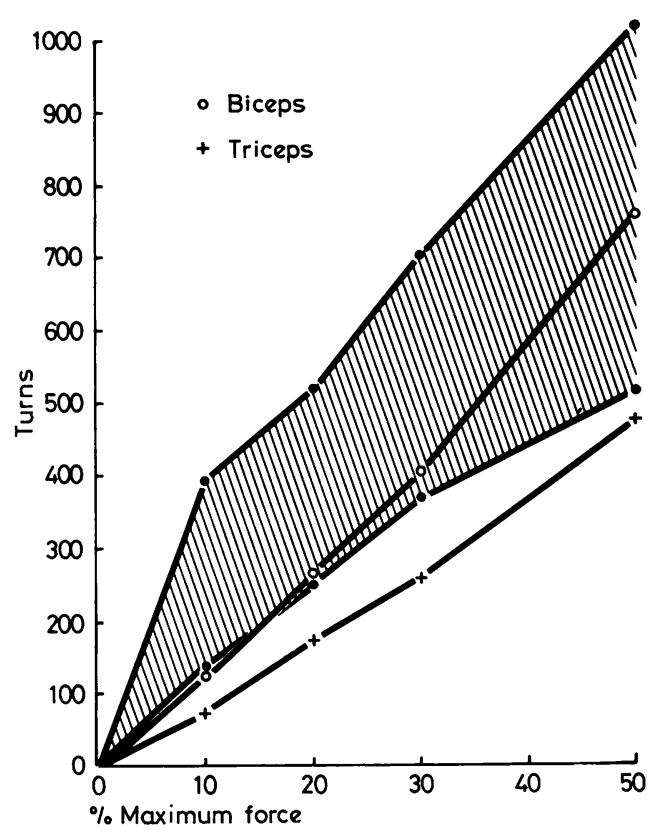

Fig 4 Patient (see text). Plot of turns against \% maximum force showing data from biceps (flexors) and reduced number in triceps (extensors) consistent with a neurogenic involvement. Control range for turns in the triceps brachii is shown (hatched area).

that is $10,20,30$, and $50 \%$ of maximum. Abnormal A/T's deviated by more than two SD's from the normal at all force levels except where the numbers in the parentheses indicate abnormal values only at those percentages of maximum force.

The patients in group 1 (table 2) were those with primary muscle disease. In all but one of these patients, turns analysis revealed consistent abnormalities, that is increased turns for the degree of force and decreased $\mathrm{A} / \mathrm{T}$ ratio. When increased turns were present, the most prominent deviation from normal was found at the lower levels force. The fractional change from normal, that is mean turns (patient) minus mean turns (control) divided by mean turns (control), was up to ten times higher at $10 \%$ maximum force in comparison to $50 \%$ (see Becker MD, fig 3). The 
Table 2 Patients: turns (T) and mean amplitude per turn $(A / T)$

\begin{tabular}{|c|c|c|c|c|}
\hline Primary muscle disease & Biceps & Brachii & Triceps & Brachi \\
\hline $\begin{array}{l}\text { Patient } \\
\text { Limb girdle dystrophy } \\
\text { Limb girdle dystrophy } \\
\text { Limb girdle dystrophy } \\
\text { Limb girdle dystrophy } \\
\text { Lmb girdle dystrophy } \\
\text { Becker muscular dystrophy } \\
\text { Dermatomyositis } \\
\text { Polymyositis } \\
\text { Myotonic dystrophy } \\
\text { Steroid myopathy } \\
\text { Myositis (scleroderma) }\end{array}$ & $\begin{array}{l}\text { T } \\
\text { inc } \\
\text { inc } \\
\text { NDA } \\
\text { inc } \\
\text { inc } \\
\text { inc } \\
\text { NDA } \\
\text { inc } \\
\text { NDA } \\
\text { NDA } \\
\text { NDA }\end{array}$ & $\begin{array}{l}\text { A/T } \\
\text { NDA } \\
\text { NDA } \\
\text { dec } \\
\text { NDA } \\
\text { dec } \\
\text { NDA } \\
\text { NDA } \\
\text { NDA } \\
\text { NDA } \\
\text { dec }(30 \%, 50 \%) \\
\operatorname{dec}(30 \%, 50 \%)\end{array}$ & $\begin{array}{l}\text { inc } \\
\text { inc } \\
\text { inc }\end{array}$ & $\begin{array}{l}\text { NDA } \\
\text { NDA } \\
\text { NDA }\end{array}$ \\
\hline $\begin{array}{l}\text { Kugelberg-Welander } \\
\text { Kugelberg-Welander } \\
\text { Kugelberg-Welander } \\
\text { Charcot-Marie-Tooth } \\
\text { Chronic renal failure } \\
\text { Remote effect carcinoma } \\
\text { III proximal muscle } \\
\quad \text { weakness etiology }\end{array}$ & $\begin{array}{l}\text { dec } \\
\text { dec } \\
\text { NDA } \\
\text { NDA } \\
\text { dec } \\
\text { dec } \\
?\end{array}$ & $\begin{array}{l}\text { inc }(10 \%, 20 \%) \\
\text { NDA } \\
\text { NDA } \\
\text { inc }(10 \%, 20 \%) \\
\text { inc } \\
\text { inc }(10 \%, 20 \%) \\
\text { NDA }\end{array}$ & $\begin{array}{l}\text { dec } \\
\text { dec }\end{array}$ & $\begin{array}{l}\text { NDA } \\
\text { NDA } \\
\text { inc } \\
\text { NDA }\end{array}$ \\
\hline
\end{tabular}

NDA—no diagnostic abnormality; inc-increased; dec-decreased

mean fractional change in the six patients in group 1 with increased turns in the biceps brachii was 2.01 at $10 \%$ maximum force while that at $50 \%$ was $0.516(\mathrm{p}<005)$. In four patients, the $A / T$ ratio was below the $95 \%$ confidence limits for normals, but this was only true at $30 \%$ and $50 \%$ of maximum force in two. In these latter two patients (steroid myopathy, scleroderma myositis), the abnormalities in the $\mathrm{A} / \mathrm{T}$ ratio were the only clear evidence of abnormality present in their total electrophysiological evaluation.

Group 2 (table 2) comprises those patients with primary neurogenic disease. Decreased turns or increased $A / T$ ratio or both were found in all but one of those patients. In comparison to the previous group, however, there was no pattern of fractional change related to the degree of force in the four patients with decrease in turns in the biceps brachii (mean fractional change at $10 \% 0 \cdot 50$, at $50 \% 0 \cdot 46$ ). An increase in the $A / T$ ratio was present in four of the patients in the biceps brachii; but, in contrast to the patients in group 1, the abnormal values were found predominently at the lower 10 and $20 \%$ maximum force.

The single patient listed in group 3 (table 2) was a 19 year old male with a long history of non-progressive proximal weakness and consistent elevations of the creatine phosphokinase up to twenty times normal. Other electrodiagnostic data revealed motor and sensory conduction studies within normal limits, normal motor unit durations in proximal muscles, and a pattern of diffuse denervation. A muscle biopsy revealed both "myopathic" and "neuropathic" changes, that is occasional atrophy and fibre groupings, internal nuclei, scattered degeneration and regeneration, some increased connective tissue and inflammatory changes. There was a decrease in turns in the biceps brachii only at $10 \%$ and $20 \%$ of maximum force while the turns in the triceps brachii were decreased at all levels of force tested (fig 4). These physiological data argued for a primary neurogenic process.

Turns analysis were performed in eight of the 18 patients in the triceps brachii (table 2). In three of the patients, this data provided the only definite abnormalities; in four, the data in the triceps brachii were comparable; and, in the remaining patient, the studies in the triceps were unrevealing while that in the biceps showed an increased $\mathrm{A} / \mathrm{T}$ ratio.

\section{Discussion}

This method of automatically analysing EMG activity during isometric voluntary contraction was adapted in our laboratory about two years ago to supplement the standard electrodiagnosis methods. Two main modifications of previous methods were made. Firstly, instead of a single fixed force at each electrode position, four different force levels corresponding to $10 \%, 20 \%$, $30 \%$ and $50 \%$ of each subject's maximum force were used. This modification was based on the assumption that all neuromuscular disorders may not affect all motor units uniformly. Secondly, two second instead of five second of EMG activity was analysed because we wanted to diminish (or exclude) any "fatigue" phenomenon and more reproducible data were obtained using the shorter sampling period.

Motor units are normally recruited in an orderly fashion according to size with the smaller, lower tension units discharging before the larger. ${ }^{7-10}$ If turns were decreased in the chronic neuropathies studied in this report, there was a relatively uniform involvement at different levels of force. This would be consistent with relatively uniform involvement of motor units of different sizes. The more prominent increase in the $\mathrm{A} / \mathrm{T}$ ratio at lower levels of muscle tension is not surprising. If motor units are increased in amplitude to a somewhat similar degree, then the method would be expected to be most sensitive in those conditions in which lower amplitude units are normally present. A high diagnostic yield for increase in mean amplitude has been renorted in patients with chronic neuropathies and no significant change in turns count. ${ }^{11}$ These studies were 
performed at a fixed level of force, and the findings are consistent with the previous observation that motor units enlarged by sprouting are less efficient than normal units. ${ }^{12}$

In contrast to neuropathies, there was a tendency for the most prominent changes in turns in myopathies to occur at the lower level of force. Although some of this could theoretically be due to overlap of motor unit firing, this is an unlikely explanation. The equipment is accurate to at least $5000 \mathrm{~Hz}$-more than twice the maximum turns per second found in any patients. Equal increases in turns at low force levels were not necessarily associated with comparable values at higher force levels (see fig 3) even if the mean amplitude per turn were within normal limits at the lower levels of force. A more reasonable explanation for the patterns of abnormal turns values in myopathies is that the initial force in the involved muscles is generated by the smaller primary or preferentially involved motor units, or both, while subsequent increases in tension are due to discharge of larger normal increment of units firing with a more normal increment of change. This is not true for all myopathies as indicated by the two patients in which turns were unremarkable but amplitude values were abnormal only at $30 \%$ and $50 \%$ of maximum force.

Analysing the data at different force levels adds to the depth and sensitivity of the method. Aside from the possibility of evaluating the effect of the disease process on motor units of different size, plotting a series of data allows a better appreciation of the physiological dysfunction present. In the patient with Becker MD shown in fig 3 , for example, a single turns value of $30 \%$ maximum force would not have revealed the more prominent abnormalities recorded at $10 \%$ and $20 \%$ maximum force levels. In three of the patients with neuropathies, the $A / T$ ratio would have been considered unremarkable if data at only $30 \%$ maximum force had been obtained. It has recently been argued that using a fixed tension, that is two or five $\mathrm{kg}$, in turns analysis is important because of the simplicity of the technirue. ${ }^{11}$ On the other hand, obtaining data at different levels of maximum force is not that much more difficult wjthont the ohvions sacrifice of sensitivity or physiological information.

In two of the patients with primary muscle disease (steroid myopathy, scleroderma myositis) turns analysis provided the only electrophysiological abnormality. In the other patients with myopathies, turns abnormalities, provided confirmation of information from more established electrodiagnostic procedures.

In the patients with neurogenic disease, one of the patients with Kugelberg-Welander disease had characteristic electrodiagnostic findings of normal conduction velocities in the presence of decreased voluntary recruitment and increased duration of motor units. In the other two patients with the same diagnosis, the clinical signs were less marked and had been present for a shorter period. In these two patients, the findings on routine motor unit evaluation were less clear while turns analysis indicated neuropathic dysfunction. Turns analysis provided the only evidence for increased motor unit size in the patient with Charcot-Marie-Tooth disease. In the remaining patients (chronic renal failure, remote effort carcinoma), evaluation of turns confirmed the basic neuropathic process despite the presence of predominantly proximal weakness. In the last patient, the aetiology had been unclear clinically, pathologically, and with routine electrodiagnostic studies. Turns evaluation clearly indicated a neurogenic process.

In three of the eight patients in which turns studies were performed in the triceps brachii, abnormalities were present although not found in comparable studies in the antagonist biceps. It therefore was evident that in some neuromuscular disorders the predilection of the disease process may not only be a question of upper as opposed to lower limb, proximal or distal, but sometimes either flexor or extensor muscles. The present method admits of easy quantification of these differences and could ultimately provide insight into the pathophvsiology of these disorders.

In conclusion, we suggest that the automatic evaluation of EMG activity during voluntary isometric contraction whether in the original form of Willison based on a constant weight, the modified version of Fuglsang-Fredericksen and coworkers analysing $30 \%$ of the maximum force, or the present modification of plotting the data at $10 \%, 20 \%, 30 \%$ and $50 \%$ of maximum force, is useful for complementing standard electrodiagnostic procedures as well as for understanding the pathophysiology of neuromuscular disorders.

We thank Dr Irwin Siegal for referring many of the patients for study. Arich Gilai, PhD, provided helpful technical suggestions.

\section{References}

1 Willison RG. Analvsis of electrical activity in healthy and dystrophic muscle in man. $J$ Neurol Neurosurg Psvchiatry 1964; 27:386-94.

2 Rose AL, Willison RG. Quantitative electromyography using automatic analysis: studies in 
healthy subjects and patients with primary muscle disease. J Neurol Neurosurg Psychiatry 1967; 30: 403-10.

3 Hayward M, Willison RG. Automatic analysis of the electromyogram in patients with partial chronic denervation. J Neurol Sci 1977; 33: 415-23.

4 Fuglsang-Frederiksen A, Masson A. Analysis of electrical activity of normal muscle in man at different degrees of voluntary effort. J Neurol Neurosurg Psychiatry 1975; 38:683-94.

5 Fuglsang-Fredericksen A, Scheel U, Buchthal F. Diagnostic yield of analysis of the pattern of electrical activity and of individual motor unit potentials in myopathy. $J$ Neurol Neurosurg Psychiatry 1976; 39:742-50.

6 Fuglsang-Fredericksen A, Scheel U, Buchthal F. Diagnostic yield of the analysis of the pattern of electrical activity of muscle and of individual motor unit potentials in neurogenic involvement. J Neurol Neurosurg Psychiatry 1977; 40:544-54.
7 Milner-Brown HS, Stein RB, Yemm R. The orderly recruitment of human motor units during voluntary isometric contraction. J Physiol (Lond) 1973; 230:359-70.

8 Tanji J, Kato M. Recruitment of motor units in voluntary contraction of a finger muscle in man. Exp Neurol 1973; 40:759-70.

9 Milner-Brown HS, Stein RB. The relationship between surface electromyogram and muscular force. J Physiol (Lond) 1975; 246:549-69.

10 Freund HJ, Budingen HJ, Dietz V. Activity of single motor units from human forearm muscles during voluntary isometric contractions. J Neurophysiol 1975; 38:933-46.

11 Hayward M. Automatic analysis of the electromyogram in healthy subjects of different ages. J Neurol Sci 1977; 33:397-413.

12 Milner-Brown HS, Stein RB, Lee RG. Contractile and electrical properties of human motor units in neuropathies and motor neuron disease. $J$ Neurol Neurosurg Psychiatry 1974; 37:670-6. 